\title{
A Comparison of Approaches to Model Uncertainty in Time Intervals
}

\section{Christophe Billiet ${ }^{1}$ José Enrique Pons $^{2}$ Olga Pons Capote ${ }^{2}$ Guy de Tré ${ }^{1}$}

\author{
${ }^{1}$ Department of Telecommunications and Information Processing, Ghent University, Belgium. \\ ${ }^{2}$ Department of Computer Science and Artificial Intelligence, University of Granada, Spain.
}

\begin{abstract}
Information systems model parts of reality by representing properties of real-world objects or concepts. As real objects or concepts often have temporal aspects, temporal notions such as time intervals are often represented. However, these may contain imperfections like uncertainties, complicating their representations. A very important purpose of information systems is to be able to query them to retrieve information, but representations of temporal notions containing uncertainties severely complicate querying. Thus, several soft computing techniques have been proposed to represent time intervals subject to uncertainties in a semantically sound way and to reason with them in a semantically sound and useful way. In the presented work, two frameworks designed for this are compared. It is found that, despite slight differences in the way these frameworks represent intervals, they provide the same results when reasoning about time intervals subject to uncertainty.
\end{abstract}

Keywords: temporal representation, temporal reasoning, possibility theory, information systems, information retrieval, ill-known intervals, uncertainty

\section{Introduction}

Information systems (IS) have always tried to model parts of reality. To achieve this modelling, IS contain data representing properties of real-world objects or concepts [1], [2]. As time is an essential aspect of many real-world objects or concepts, IS often contain data representing temporal notions which describe such temporal properties [3], [4]. Such temporal notions usually take the form of either instants [5], which can informally be seen as infinitely short 'moments' or 'points' in time, or time intervals [5].

Data are often produced by humans, but human-made data are prone to imperfections: some data may be vague, imprecise, incomplete, contradictory or uncertain. Data representing temporal notions may contain such imprecisions too [1], [2], [4], [6], [7]. Although The work presented in this paper is specifically concerned with time intervals (and as a special case: instants) subject to uncertainty.
Generally, one of the most important purposes of an IS is to allow the retrieval of information or knowledge deduced from its data. Such retrieval is usually done by querying the IS and examining or analyzing the query results or by visualizing the contents of the IS, querying this visualization and examining or analyzing the resulting visualization(s).

Of course, when temporal information is represented in an IS, querying this IS may have a temporal aspect too. Usually, querying such an IS is conceptually done by specifying one or more time indications and requesting information that is in a specific relationship with these indications, where the semantics of these relationships are specifically temporal [1], [2], [8]. Thus, some existing proposals have considered groups of basic relationships between time indications used to construct and express specific temporal relationships [9], [10], [11]. Notably, Allen [12] presented a reasoning framework containing all semantically useful basic temporal relationships between time intervals (and as a special case instants) and subsequently used these in a framework to represent and reason with timerelated actions [13] and compared them with other approaches [14]. The resulting relationships are shown in figure 1. These Allen relationships are used in the presented work.

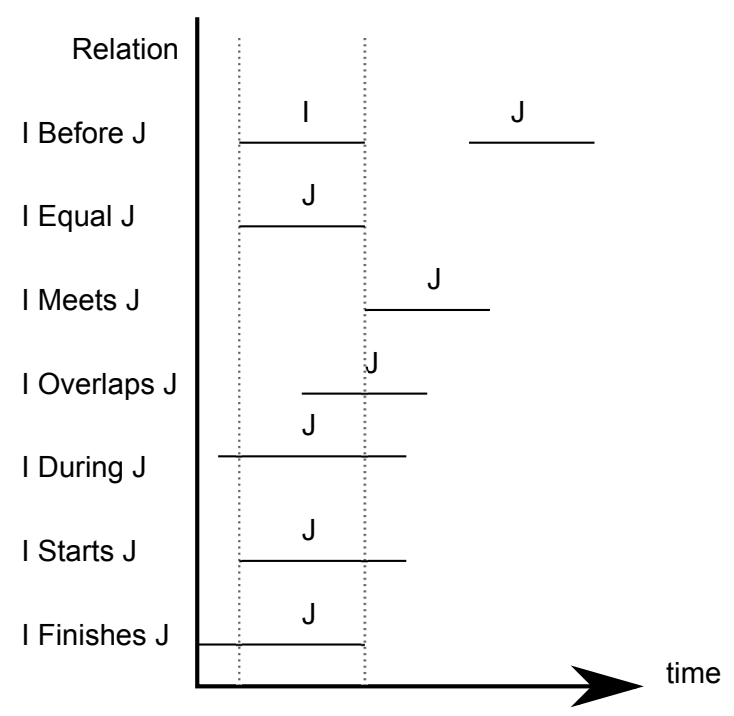

Figure 1: The Allen relationships between two crisp intervals $I$ and $J$. 
To be able to query IS containing data representing time indications subject to uncertainty, a framework is necessary, able to represent uncertainties in time indications in a semantically sound way, without (much) information loss and able to temporally reason with such time indications in a semantically sound and useful way [7], [11], [15]. Although more proposals for such frameworks exist, the work presented in this paper focusses on just two: the ill-known constraint (IKC) framework [16] and the triangular model (TM) framework [17].

The work presented in this paper consists of a comparison of both frameworks, about the approaches they use to represent time intervals subject to uncertainty and to reason about the temporal relationships between such intervals and time intervals without uncertainty.

The structure of this paper is as follows: section 2 presents some general preliminaries and naming and notation conventions used in this paper. Sections 3 and 4 introduce the IKC and TM frameworks respectively: both sections first introduce some specific preliminary concepts and techniques, then explain how the representation of time intervals by the framework is done and finally show how the evaluation of Allen relationships between an interval subject to uncertainty and one without uncertainty is done. In section 5 , both frameworks are compared: first their approaches to representing time intervals are compared, next their approaches to evaluating Allen relationships. Also, an example is given. Finally, section 6 presents the principal conclusions of the presented work and some possible future work.

\section{General Preliminaries, Notations and Nomenclature}

Often, time is thought of as following an axis (a time axis) and thus it is often seen as an ordered set of infinitely short 'moments' or 'points' in time, called instants [5]. This is also the case in the presented work. A time interval is then an interval subset of this ordered set of instants. In this paper, instants are noted using lowercase letters and time intervals using uppercase letters. Also, a time interval $I$ starting at (and containing) instant $s$ and ending at (and containing) instant $e$ is noted using square brackets: $I=[s, e]$.

The presented work allows time intervals to be subject to uncertainties. These uncertainties are always seen as caused by a (partial) lack of knowledge: the exact, intended time interval is not known, eventhough there is only one correct intended time interval and as such no variability. Confidence about exactly which interval is the intended one in the context of such uncertainties is modelled using possibility theory [15]. In the presented work, possibility is always interpreted as plausibility given a (partial) lack of knowledge.

To be able to distinguish easily, time intervals not subject to uncertainties will be called crisp time intervals (CTI) in the presented work.

\section{The Ill-known Constraint Framework}

In [16], the ill-known constraint framework is introduced. In this section, its main aspects concerning interval representation and Allen relationship evaluation are briefly presented.

\subsection{Preliminary Concepts}

The IKC framework relies on the concepts of possibilistic variables, ill-known values and ill-known intervals [1], [2], [15], [16]. These are introduced below.

Definition 1. A possibilistic variable $X$ on a universe $U$ is defined as a variable taking exactly one value in $U$, but for which this value is (partially) unknown. The variable $X$ 's possibility distribution $\pi_{X}$ on $U$ models the available knowledge about the value that $X$ takes: for each $u \in U, \pi_{X}(u)$ represents the possibility that $X$ takes the value $u$.

As mentioned above, possibility is interpreted as plausibility in the presented work and thus the possibility that possibilistic variable $X$ on $U$ takes the value $u \in U$ is interpreted as a measure of how plausible it is that $X$ takes the value $u$, given (partial) knowledge about the value $X$ takes.

Now consider a set $U$ containing single values (and not collections of values). When a possibilistic variable $X_{v}$ is defined on such a set $U$, the unique value $X_{v}$ takes, which is (partially) unknown, will be a single value in $U$ and is called an ill-known value (IKV) in $U$ in this work [2], [16], [18].

In this paper, possibilistic variables will be denoted using uppercase letters and IKV using lowercase letters.

\subsection{Representation of Time Intervals Subject to Uncertainty}

The IKC framework allows uncertainty in intervals by supporting a kind of ill-known intervals $[1],[2],[15],[16]$ :

Definition 2. Consider an ordered set $U$. An $i l l$ known interval (IKI) in $U$ is an interval in $U$ of which both boundary values are ill-known values in $U$.

Specifically with regards to time, an IKI in an ordered set of instants representing time is called an ill-known time interval (IKTI) in the presented work. In this paper, an IKTI with starting instant $s$ and ending instant $e$ will be noted $[s, e]$.

\subsection{Evaluation of Allen Relationships}

At the base of the framework's approach to evaluating Allen relationships are ill-known constraints [16]: 
Definition 3. Given an ordered set $U$, an ill-known constraint (IKC) $C$ on $U$ is specified by means of a binary relation $R \subseteq U^{2}$ and a fixed IKV $v$ in $U$ defined by its possibilistic variable $V$ on $U$, i.e.:

$$
C \triangleq(R, v)
$$

Any set $A \subseteq U$ now satisfies $\operatorname{IKC} C=(R, v)$ if and only if:

$$
\forall a \in A:(a, v) \in R
$$

The satisfaction of an IKC $C$ by a set $A$ will be noted $C(A)$ in this paper.

Consider an ordered set $U$, an $\operatorname{IKC} C \triangleq(R, v)$ on $U$ and a set $A \subseteq U$. Due to the uncertainty about the exact value of $v$, it is uncertain whether $A$ satisfies $C$ or not. Confidence about the plausibility that $A$ satisfies $C$ can be expressed using a possibility degree $\operatorname{Pos}(C(A))$ and a necessity degree $\operatorname{Nec}(C(A))$, which are shown to be calculated as follows:

$$
\begin{aligned}
& \operatorname{Pos}(C(A))=\min _{a \in A}\left(\sup _{(a, w) \in R} \pi_{V}(w)\right) \\
& \operatorname{Nec}(C(A))=\min _{a \in A}\left(\inf _{(a, w) \notin R} 1-\pi_{V}(w)\right)
\end{aligned}
$$

Given an ordered set $U$, possibility and necessity degrees expressing confidence about the plausibility that a set $A \subseteq U$ satisfies a boolean aggregation of IKC on $U$ can be found by using the possibilistic extensions of boolean operators 'and' $(\wedge)$, 'or' $(\vee)$ and 'not' $(\neg)$ and can be calculated using the following expressions:

$$
\begin{array}{r}
\operatorname{Pos}\left(\left(C_{1}(A)\right) \wedge \ldots \wedge\left(C_{n}(A)\right)\right)=\min _{i=1 \ldots n}\left(\operatorname{Pos}\left(C_{i}(A)\right)\right) \\
\operatorname{Nec}\left(\left(C_{1}(A)\right) \wedge \ldots \wedge\left(C_{n}(A)\right)\right)=\min _{i=1 \ldots n}\left(\operatorname{Nec}\left(C_{i}(A)\right)\right) \\
\operatorname{Pos}\left(\left(C_{1}(A)\right) \vee \ldots \vee\left(C_{n}(A)\right)\right)=\max _{i=1 \ldots n}\left(\operatorname{Pos}\left(C_{i}(A)\right)\right) \\
\operatorname{Nec}\left(\left(C_{1}(A)\right) \vee \ldots \vee\left(C_{n}(A)\right)\right)=\max _{i=1 \ldots n}\left(\operatorname{Nec}\left(C_{i}(A)\right)\right) \\
\operatorname{Pos}(\neg C(A))=1-\operatorname{Nec}(C(A)) \\
\operatorname{Nec}(\neg C(A))=1-\operatorname{Pos}(C(A))
\end{array}
$$

Here, $C$ and all $C_{i}$ for which $i \in \mathbb{N}_{0}$ and $1 \leq i \leq n$ denote IKC on $U$.

The IKC framework now allows evaluating a given Allen relationship $A R$ between a given CTI $I$ and a given IKTI $J=[s, e]$ by allowing to calculate the possibility and necessity that $I A R J$ holds. For this, the combination of $A R$ and $J$ is translated to a specific boolean aggregation of specific IKC. These translations are shown in table 1 . Every row of this table corresponds to an Allen relationship. The Allen relationships the rows correspond to, are shown in the 'Allen Relationship' column, the collections of specific IKC for given Allen relationships are shown in the 'Constraints' column (every $C_{i}, i \in\{1,2,3,4\}$ denotes an IKC) and the specific aggregation of these IKC used for evaluation of the Allen relationships are shown in column 'Aggregation'. Finally, the possibility and necessity degrees expressing confidence about the plausibility that $I A R J$ holds are then the possibility and necessity expressing confidence about the plausibility that $I$ satisfies the specific aggregation of specific IKC found as translation of the combination of $A R$ and $J$.

Using the formulas shown above, the requested possibility and necessity degrees can be calculated from these.

\begin{tabular}{|c|c|c|}
\hline Allen Relationship & Constraints & Aggregation \\
\hline I before $\mathrm{J}$ & $C_{1} \triangleq(<, s)$ & $C_{1}(I)$ \\
\hline \multirow{3}{*}{ I equal J } & $C_{1} \triangleq(\geq, s)$ & $C_{1}(I) \wedge$ \\
& $C_{2} \triangleq(\neq, s)$ & $\neg C_{2}(I) \wedge$ \\
& $C_{3} \triangleq(\leq, e)$ & $C_{3}(I) \wedge$ \\
& $C_{4} \triangleq(\neq, e)$ & $\neg C_{4}(I)$ \\
\hline \multirow{2}{*}{ I meets J } & $C_{1} \triangleq(\leq, s)$ & $C_{1}(I) \wedge$ \\
& $C_{2} \triangleq(\neq, s)$ & $\neg C_{2}(I)$ \\
\hline \multirow{2}{*}{ I overlaps $\mathrm{J}$} & $C_{1} \triangleq(<, e)$ & $C_{1}(I) \wedge$ \\
& $C_{2} \triangleq(\leq, s)$ & $\neg C_{2}(I) \wedge$ \\
& $C_{3} \triangleq(\geq, s)$ & $\neg C_{3}(I)$ \\
\hline & $C_{1} \triangleq(>, s)$ & $\left(C_{1}(I) \wedge\right.$ \\
I during J & $C_{2} \triangleq(\leq, e)$ & $\left.C_{2}(I)\right) \vee$ \\
& $C_{3} \triangleq(\geq, s)$ & $\left(C_{3}(I) \wedge\right.$ \\
& $C_{4} \triangleq(<, e)$ & $\left.C_{4}(I)\right)$ \\
\hline \multirow{2}{*}{ I starts J } & $C_{1} \triangleq(\geq, s)$ & $C_{1}(I) \wedge$ \\
& $C_{2} \triangleq(\neq, s)$ & $\neg C_{2}(I)$ \\
\hline \multirow{2}{*}{ I finishes J } & $C_{1} \triangleq(\leq, e)$ & $C_{1}(I) \wedge$ \\
& $C_{2} \triangleq(\neq, e)$ & $\neg C_{2}(I)$ \\
\hline
\end{tabular}

Table 1: The translations of Allen relationships to the IKC framework.

\section{The Triangular Model Framework}

In [19], the TM framework for crisp intervals is introduced, based on [20]. In [17], the framework is generalized to allow the representation of and reasoning with time intervals subject to uncertainty. In this section, this generalization's main aspects towards interval representation and Allen relationship evaluation are briefly presented.

\subsection{Preliminary Concepts}

In the triangular model framework, the approaches to interval representation and Allen relationship evaluation are closely related to the approach to interval visualization. Usually, different time intervals are visualized as different parallel line segments in the same image plane. However, as this linear approach introduces a few issues, time intervals are visualized as points in the image plane in the TM framework [17], [19]. To achieve this, a horizontal line segment visualizing the used (part of the) time 
axis is drawn in the image plane (this is called the time line). Then, a triangle is drawn, using this line segment as a side of which the angles with the other two sides have sizes $\alpha$ and $-\alpha$ respectively. The area contained in this triangle is called the interval space and will contain all visualizations of intervals. Now, the procedure used to visualize a CTI $[s, e]$ in the interval space is the following [17], [19]:

1. $s$ is visualized as a point on the time line.

2. A straight half-line $L_{s}$ is constructed from this point, having, in this point, an angle of size $\alpha$ with the time line.

3. $e$ is visualized as a point on the time line.

4. A straight half-line $L_{e}$ is constructed from this point, having, in this point, an angle of size $-\alpha$ with the time line.

5. The intersection point of $L_{s}$ and $L_{e}$ is the intended visualization of interval $[s, e]$.

A similar point visualizing an interval is generally called an interval point [17], [19]. In this work, as it is usually done, the size of $\alpha$ is chosen to be $45^{\circ}$. An example of such construction is shown in figure 2 .

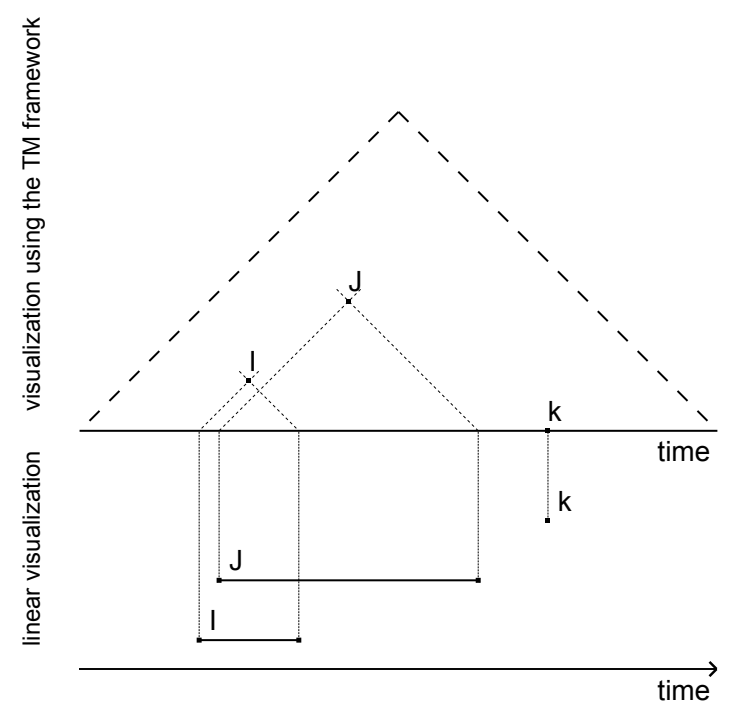

Figure 2: The visualization of several CTI using the TM framework.

\subsection{Representation of Time Intervals Subject to Uncertainty}

The TM framework allows uncertainty in time intervals by supporting uncertain time intervals [17]:

Definition 4. Given an ordered set of instants $T$, an uncertain time interval (UTI) $J$ in $T$ is here a time interval defined by a pair

$$
J \triangleq\left(\pi_{s}, \pi_{e}\right)
$$

where $\pi_{s}$ and $\pi_{e}$ are two convex [15] possibility distributions on $T$.

Consider an ordered set of instants $T$ and an UTI $J$ in $T$, defined by the pair $\left(\pi_{s}, \pi_{e}\right)$. Given an instant $x \in T, \pi_{s}(x)$ now expresses the possibility that $x$ is the starting instant of $J$ and $\pi_{e}(x)$ expresses the possibility that $x$ is the ending instant of $J$. Thus, $J$ intends to indicate just one CTI, but it is (partially) unknown exactly which time interval this is. The possibility $\pi_{J}(I)$ that a given CTI $I=\left[s_{i}, e_{i}\right]$ is the exact time interval intended by $J$ can now be uniquely determined by [17]:

$$
\pi_{J}(I)= \begin{cases}\min \left(\pi_{s}\left(s_{i}\right), \pi_{e}\left(e_{i}\right)\right), & \text { if } s_{i} \leq e_{i} \\ 0, & \text { otherwise }\end{cases}
$$

Now consider an ordered set of instants $T$ and a visualization of this in an image plane, using the TM framework. An UTI $J$ in $T$ defined by a pair $\left(\pi_{s}, \pi_{e}\right)$ is now visualized as an area in the image plane. This area is constructed as follows: for every CTI $K$ for which the possibility $\pi_{J}(K)$ of $K$ being the CTI intended by $J$ is higher than zero, the interval point is drawn and is given a color. This color is part of a linear single-color greyscale and its saturation is linearly related to the degree of possibility that $K$ is the CTI intended by $J$. Together, these drawn interval points form the visualization of $J[17]$. An example of such construction is shown in figure 3 . In this figure, a darker color of interval point indicates a higher and a lighter color a lower possibility that the corresponding CTI is the intended interval.

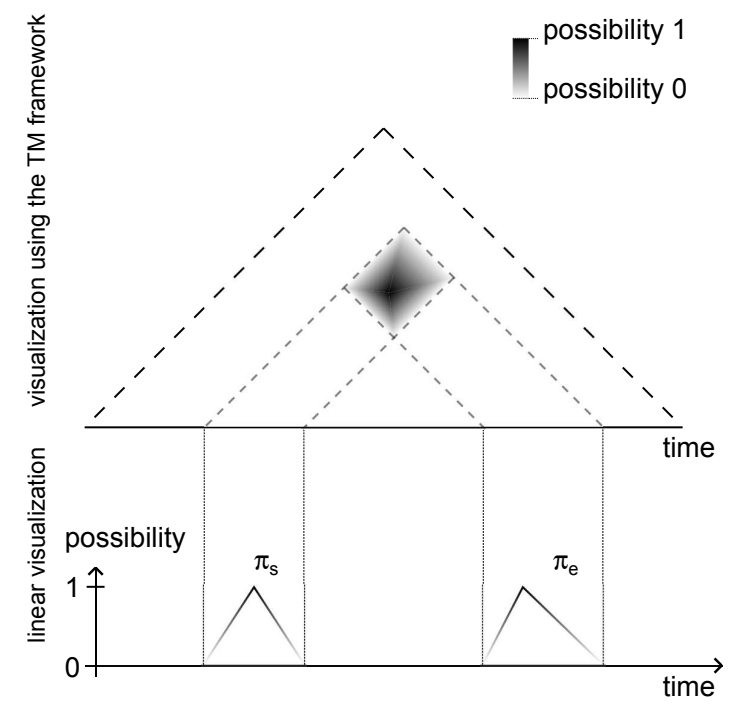

Figure 3: The visualization of one UTI defined by a pair $\left(\pi_{s}, \pi_{e}\right)$ using the TM framework.

\subsection{Evaluation of Allen Relationships}

At the base of the framework's approach to evaluating Allen relationships is the concept of uncertain relational zones [17]:

Definition 5. For a given UTI $J$, the uncertain relational zones (URZ) are fixed areas in the interval space.

Now, given an UTI $J$ and its visualization in an interval space, the procedure to visualize its URZ is the following [17]: 
1. The visualizations (the points) on the time line of both the smallest and greatest instant with a non-zero possibility of being the starting instant of $J$ are determined.

2. Two straight half-lines are constructed from each of these points: one having, in the point, an angle of size $\alpha$ with the time line and one having, in the point, an angle of size $-\alpha$ with the time line.

3. The visualizations (the points) on the time line of both the smallest and greatest instant with a non-zero possibility of being the ending instant of $J$ are determined.

4. Two straight half-lines are constructed from each of these points: one having, in the point, an angle of size $\alpha$ with the time line and one having, in the point, an angle of size $-\alpha$ with the time line.

5. The lines constructed this way divide the interval space in at most fifteen different areas, including the area corresponding to the visualization of $J$ itself. These areas are the intended visualizations of $J$ 's URZ.

In this procedure, $\alpha$ is the same angle size as in the procedure for the visualization of CTI. The results of this procedure for an example UTI $J$ (as wel as the visualization of $J$ itself) are shown in figure 4.

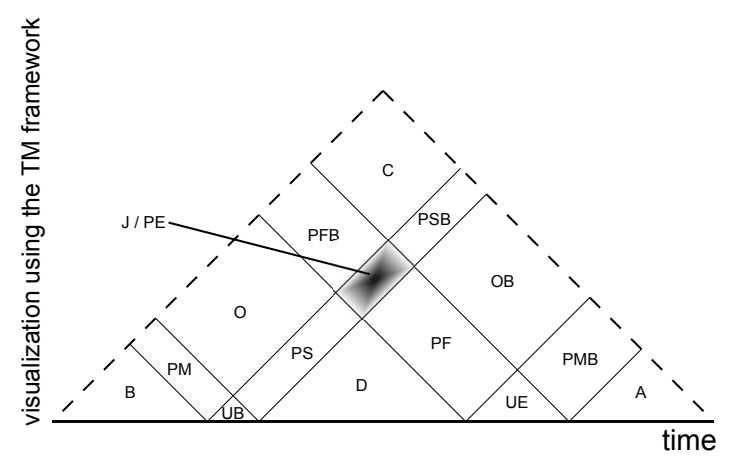

Figure 4: The visualization of the URZ for a given UTI $J$, using the TM framework.

Given an UTI $J$ and its URZ, every URZ is assigned a symbol and a name and corresponds to a set of Allen relationships [17]. These symbols and names are used to uniquely identify each of J's URZ. The set of Allen relationships corresponding to an URZ contains every Allen relationship in which any interval visualized by one of the URZ's interval points could be with the interval intended by $J$, depending on exactly which CTI $J$ actually intends to represent. In figure 4, the URZ's symbols are noted inside the areas visualizing their respective URZ's. In table 2, every different row corresponds to a different URZ. In the first two columns, the symbol and name of the URZ are given, the last column contains the set of corresponding Allen relationships. For these, the following notations are used:
- 'E' denotes 'equals'.

- 'S' denotes 'starts'.

- 'SB' denotes 'started by'.

- ' $F$ ' denotes 'finishes'.

- 'FB' denotes 'finished by'.

- 'M' denotes 'meets'.

- 'MB' denotes 'met by'.

- 'O' denotes 'overlaps'.

- 'OB' denotes 'overlapped by'.

- 'D' denotes 'during'.

- 'C' denotes 'contains'.

- 'B' denotes 'before'.

- 'A' denotes 'after'.

\begin{tabular}{|l|l|l|}
\hline Symbol & Name & Relationships \\
\hline B & Before & B \\
O & Overlaps & O \\
C & Contains & C \\
D & During & D \\
OB & Overlapped By & OB \\
A & After & A \\
PM & Possibly Meets & M, B, O \\
PS & Possibly Starts & O, S, D \\
PFB & Possibly Finished By & O, FB, C \\
PE & Possibly Equal & E, FB, SB, F, \\
PSB & Possibly Started By & S, C, D, O, OB \\
PF & Possibly Finishes & OB, F, D \\
PMB & Possibly Met By & OB, MB A \\
UB & Uncertain Beginning & B, M, O, S, D \\
UE & Uncertain Ending & D, F, OB, MB, A \\
\hline
\end{tabular}

Table 2: The fifteen possible URZ for a given UTI.

The TM framework now allows evaluating the Allen relationships a given CTI $I=[s, e]$ could be in with a given UTI $J$, depending on the exact CTI intended by $J$. This is done as follows. First, $J$ and its URZ are visualized in an interval space. Next, $I$ 's interval point, its construction half-lines $L_{s}$ and $L_{e}$ and the visualization of its starting and ending instants on the time line are drawn in the same interval space. Then, evaluation is a matter of position (in the following, the possibility that $I$ is the interval intended by $J$ is denoted $\pi_{J}(I)$ and the possibility that $I$ is in Allen relationship $A R$ with $J$ is denoted $\operatorname{Pos}(I A R J))$ [17]:

- if $I$ 's interval point is located in an URZ corresponding with a singleton of Allen relationships $\{R\}$, it is in relationship $R$ with the interval intended by $J$, whichever this interval is. Thus: $\operatorname{Pos}(I A R J)=1$.

- if $I$ 's interval point is located in an URZ corresponding to a non-singleton set of $n$ Allen relationships $\left\{R_{1}, R_{2}, \ldots, R_{n}\right\}$, two more half-lines are drawn: one (denoted $L_{s}^{\prime}$ ) from the visualization of $s$ on the time line, having, in this point, an angle of size $-\alpha$ with the time line and one (denoted $L_{e}^{\prime}$ ) from the visualization of $e$ on the time line, having, in this point, an angle of size $\alpha$ with the time line. Now, some of 
these half-lines $L_{s}, L_{s}^{\prime}, L_{e}$ and $L_{e}^{\prime}$ may intersect with the area visualizing $J$. These intersection line segments will divide the area visualizing $J$ in $n$ area parts $p_{i}, 1 \leq i \leq n$ (intersection line segments also count as area parts), each containing only interval points visualizing intervals with a non-zero possibility of being the interval intended by $J$. Each of these parts $p_{i}$ will now correspond to an Allen relationship $R_{i}$. Now, for each $R_{i}$ :

$$
\operatorname{Pos}\left(I R_{i} J\right)=\sup _{K \in p_{i}} \pi_{J}(K)
$$

Here, the intervals $K$ are CTI and the expression ' $K \in p_{i}$ ' is a notation to express that $K$ is visualized by an interval point contained in area part $p_{i}$.

\section{Comparison of Both Frameworks}

In this section, first the approaches of the IKC and TM frameworks towards interval representation are compared. Next, their approaches towards the evaluation of Allen relationships between a crisp interval and an interval subject to uncertainty are compared.

\subsection{Comparison of Approaches to Interval Representation}

The IKC framework uses IKTI to represent time intervals subject to uncertainty, whereas the TM framework uses UTI for this. In both approaches, to consider uncertainty about the exact CTI which is intended, uncertainty about the exact starting and ending instants of the intended interval is considered and confidence in the context of this uncertainty is expressed using two possibility distributions. In an IKTI, these possibility distributions each define one IKV. One of these then defines the IKTI's starting instant and the other defines its ending instant. In an UTI, one of these possibility distributions is directly meant to define the UTI's starting instant and the other is directly meant to define the UTI's ending instant. It is obvious that the concepts of IKTI and UTI are exactly the same, except for the explicit usage of the concept of IKV in IKTI to define and describe uncertainty about starting and ending instants. This equality implies that both approaches have the same basic restriction: they cannot represent every kind of interval subject to uncertainty imaginable. For example, imagine an ordered set of instants coinciding with $\mathbb{N}_{0}$ and imagine an interval subject to uncertainty in this set, where the intended interval is either $[2,4]$ (possibility 1 ) or $[3,7]$ (possibility 1 ). This interval can not be modelled by a combination of possibility distributions defining the starting and ending instants without making $[2,7]$ and $[3,4]$ also possible as intended intervals to some extent. This issue was first suggested about IKTI in [2].

Now, a major difference between the two frameworks, concerning their approaches towards the rep- resentation of time intervals in general, is that the TM framework includes visualization in its approach.

A first consequence of this is that the TM framework allows the visualization of multiple CTI in the same image plane. In theory, it should also allow the visualization of multiple UTI in the same image plane. However, if the interval space area's visualizing these UTI overlap, it is not yet researched which greyscale (or other) color and intensity each interval point in this overlapping area should have, as the appearance of such interval point should both reflect the possibility of it being the interval intended by the one UTI and the possibility of it being the interval intended by the other UTI. The advantage of the ability to visualize multiple time intervals in the same image plane is that a human observer could easily assess certain characteristics of a distribution of time intervals from an image containing their visualization. The IKC framework, on the other hand, doesn't incorporate any form of visualization and doesn't allow such human assessments.

A second consequence of this is that the TM framework requires that an UTI can be visualized, using a visualization method which actually visualizes the possibility of a CTI of being the interval intended by this UTI for every CTI that has a nonzero possibility of this. Thus, a method is required, to calculate the possibility of a given CTI of being the interval intended by an UTI based on the possibility distributions defining this UTI. This method is found by demanding that the given CTI's starting instant is the intended interval's starting instant and that the given CTI's ending instant is the intended interval's ending instant and by determining the possibility of the conjunction of both demands using the standard possibility theory conjunction operator 'minimum'. Although not necessary for the correct and consistent functioning of the TM framework, it appears to be the intention of the TM framework that possibility about the exact starting or ending instant of the interval intended by the UTI could be derived from the possibility distribution defining the possibility that a given CTI is the interval intended by the UTI. For this derivation to be consistent, the possibility distributions defining the UTI must be convex [15]. Given an ordered set $T$ and an UTI $J=\left(\pi_{J_{s}}, \pi_{J_{e}}\right)$ in $T$ with possibility $\pi_{J}(I)$ that a given CTI $I=\left[s_{i}, e_{i}\right]$ is the exact time interval intended by $J$, the derivations can be calculated as follows:

$$
\begin{aligned}
& \pi_{J_{s}}\left(s_{i}\right)=\max _{K=\left[s_{i}, k\right], k \in T, k>s_{i}}\left(\pi_{J}(K)\right) \\
& \pi_{J_{e}}\left(e_{i}\right)=\max _{K=\left[k, e_{i}\right], k \in T, k<e_{i}}\left(\pi_{J}(K)\right)
\end{aligned}
$$

With respect to this convexity demand, the IKC framework is similar: the possibility distributions defining the starting and ending IKV of an IKTI are also demanded to be convex by the IKC framework, although this appears not to be necessary for the 
framework to function correctly and consistently.

\subsection{Comparison of Approaches to Allen Relationship Evaluation}

As mentioned before, a major difference between the two frameworks, concerning their approaches towards the representation of time intervals in general, is that the TM framework includes visualization in its approach. As a result, it also includes visualization in its approach towards the evaluation of Allen relationships between a CTI and an UTI.

A first consequence of this is that, given a CTI, an UTI, its URZ and their visualizations in the same image, the TM framework allows a visual, human assessment of the degree of possibility of this CTI being in an Allen relationship with this UTI, for every Allen relationship with a non-zero such degree, based on this image. Moreover, those with possibility degree equal to zero can be easily found before any calculation is done: they are the relationships not contained in the set corresponding to the URZ containing the CTI's interval point. On the other hand, to examine the respective possibilities of a given CTI of being in several different Allen relationships with a given IKTI using the IKC framework, a new collection of specific IKC and a specific aggregation of these should be constructed for every Allen relationship under consideration, allowing to calculate its exact possibility and necessity. In contrast to the TM framework, using the IKC framework a human assessment before any calculation is not possible and it is not known before any calculation which Allen relationships will result in a possibility degree equal to zero.

A second consequence is that, given an UTI and its URZ, multiple CTI can be visualized in the same image as the UTI and its URZ. Thus, the same image could provide a visual, human assessment of the possibilities with which multiple CTI are in Allen relationships with the UTI, before any calculation is done. Again, the IKC framework would need a different collection of specific IKC and a specific aggregation of these for every Allen relationship under consideration, but calculating the possibilities for several CTI to be in a given Allen relationship with a given IKTI would not provide much extra work.

A third consequence is that, in the TM framework, given an UTI, its URZ and a visualization of these in the same image, a given Allen relationship could correspond with several URZ. Thus, given a distribution of CTI, it is not easy to visually assess which CTI have a non-zero possibility of being in the given Allen relationship with the given UTI. On the other hand, assessing this using the IKC framework is impossible without any calculation, but the necessary calculations are pretty straightforward.

Although this has not been rigorously researched yet, it is the conviction of the authors that a great strength of the IKC framework lies in its modular approach towards the evaluation of temporal relationships, resulting in a flexibility and an easy handling of complex temporal relationships, while the visualization of these using the TM framework could become complex and heavy. This would give the IKC framework a major advantage over the TM framework, when used in reasoning systems like e.g. decision support systems. Moreover, in some cases the visualization step used in the TM framework could be a redundant step.

\subsection{An Example}

In this section, some of the comparison results presented above will be illustrated using a simple example.

Example 1. Consider an ordered set of instants $T$ coinciding with $\mathbb{R}$, a CTI $I=[1,3]$ and a time interval $J$ of which the starting instant is defined by a triangular possibility distribution on $T$ with core $\{5\}$ and support $[2,7]$ and of which the ending instant is defined by a triangular possibility distribution on $T$ with core $\{12\}$ and support $[9,15]$. In a real-life situation, such distributions could be decided by experts or deduced from existing evidence. The visualization of this example situation using the TM framework is shown in figure 5. The interval point for $I$ lies in the URZ 'PM'. Thus, possibility is higher than zero for $I$ to be in a 'meets', 'before', or 'overlaps' relationship with $J$. As the darkest point in the 'overlaps' area part is very light, the darkest point in the 'meets' line segment is of almost exactly the same lightness and the darkest point in the 'before' area part is perfectly black, it can be estimated that $I$ overlaps $J$ with a low possibility, $I$ meets $J$ with the same low possibility and $I$ is before $J$ with possibility 1 . Calculation using the IKC framework now learns that:

$$
\begin{aligned}
\operatorname{Pos}(\text { Ioverlaps } J) & =\min (1,1 / 3,1)=1 / 3 \\
\operatorname{Pos}(\text { Imeets } J) & =\min (1,1 / 3)=1 / 3 \\
\operatorname{Pos}(\text { Ibefore } J) & =\min (1)=1
\end{aligned}
$$

\section{Conclusions}

In this paper, a comparison is presented between two different frameworks designed to represent time intervals subject to uncertainty and evaluate temporal relationships between such intervals and crisp intervals: the triangular model (TM) framework and the ill-known constraint (IKC) framework. It is concluded that:

- With respect to representation, both frameworks differ only slightly, with the TM framework allowing easier human assessments, due to its approach including visualization.

- With respect to temporal relationship evaluation, the TM framework allows easy human assessments in several situations, but the IKC framework seems more fitted for complex reasoning, due to its modular build. 


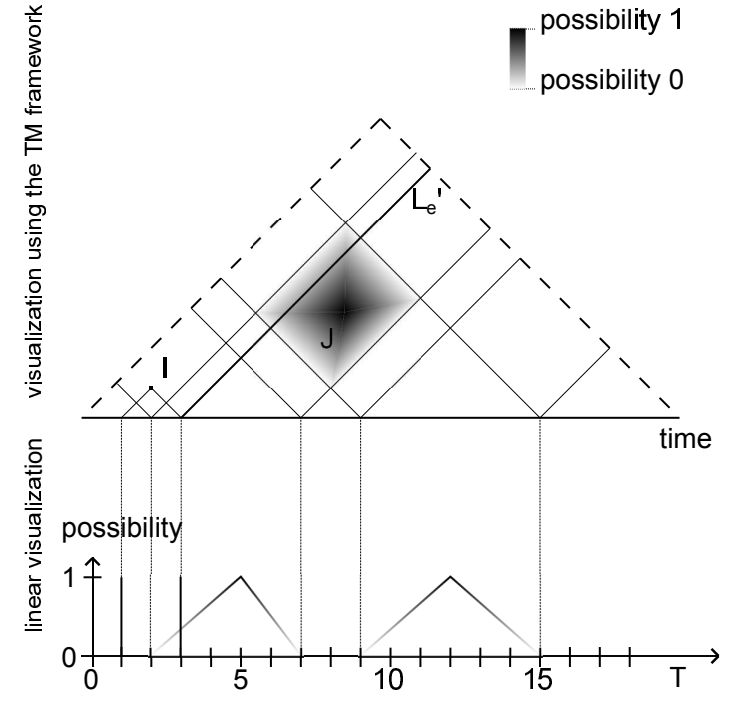

Figure 5: The visualization of the example using the TM framework.

Future research will deal with the evaluation of temporal relationships between several time intervals subject to uncertainty and with querying aspects in both frameworks.

\section{Acknowledgements}

Part of the research is supported by the grant BES2009-013805 within the research project TIN200802066: Fuzzy Temporal Information treatment in relational DBMS.

\section{References}

[1] J. E. Pons, C. Billiet, O. Pons Capote, and G. De Tré. A possibilistic valid-time model. In Communications in Computer and Information Science, volume 297, pages 420-429, 2012.

[2] C. Billiet, J. E. Pons, O. Pons Capote, and G. De Tré. Evaluating possibilistic valid-time queries. In Communications in Computer and Information Science, volume 297, pages 410 419, 2012.

[3] A. Bolour, T. L. Anderson, L. J. Dekeyser, and H. K. T. Wong. The role of time in information processing: a survey. ACM SIGMOD Record, 12(3):27-50, 1982.

[4] B. Van der Cruyssen, R. De Caluwe, and G. De Tré. A theoretical fuzzy time model based on granularities. EUFIT'97, pages $1127-$ 1131, 1997.

[5] M. H. Böhlen et al. The Consensus Glossary of Temporal Database Concepts-February 1998 Version. In Lecture Notes in Computer Science, volume 1399/1998, pages 367-405, 1998.

[6] F. Devos, N. Van Gyseghem, R. Vandenberghe, and $R$. De Caluwe. Modelling vague lexical time expressions by means of fuzzy set theory. Journal of Quantitative Linguistics, 1(3):189 194, 1994.
[7] D. Dubois, A. Hadjali, and H. Prade. Fuzziness and uncertainty in temporal reasoning. Journal of Universal Computer Science, 9(9):11681194, 2003.

[8] J. E. Pons, N. Marín, O. Pons, C. Billiet, and G. De Tré. A relational model for the possibilistic valid-time approach. International Journal of Computational Intelligence Systems, 5(6):1068-1088, 2012.

[9] J. M. Medina, O. Pons, and M. Amparo Vila. Gefred. a generalized model of fuzzy relational databases. Information Sciences, 76:87-109, 1994.

[10] S. Schockaert, M. De Cock, and E. E. Kerre. Fuzzifying allen's temporal interval relations. Fuzzy Systems, IEEE Transactions on, 16(2):517 -533, 2008.

[11] D. Dubois, A. Hadjali, and H. Prade. A Possibility Theory-Based Approach to the Handling of Uncertain Relations between Temporal Points. International Journal of Intelligent Systems, 22(2):157-179, 2007.

[12] J. F. Allen. Maintaining knowledge about temporal intervals. Communications of the ACM, 26(11):832-843, 1983.

[13] J. F. Allen. Towards a General Theory of Action and Time. Artificial Intelligence, 23(2):123-154, 1984.

[14] J. F. Allen. Time and time again: The many ways to represent time. International Journal of Intelligent Systems, 6(4):341-355, 1991.

[15] D. Dubois and H. Prade. Ranking fuzzy numbers in the setting of possibility theory. Information Sciences, 30(3):183-224, 1983.

[16] J E. Pons, A. Bronselaer, G. De Tré, and O. Pons. Possibilistic evaluation of sets. International Journal of Uncertainty, Fuzziness and Knowledge-Based Systems, 2012. Accepted for publication.

[17] G. De Tré et al. Visualising and handling uncertain time intervals in a two-dimensional triangular space. In Proceedings of the 2nd World Conference on Soft Computing, pages 585-592, 2012.

[18] D. Dubois and H. Prade. Incomplete conjunctive information. Computers \& Mathematics with Applications, 15(10):797-810, 1988.

[19] N. Van de Weghe, R. Docter, P. De Maeyer, B. Bechtold, and K. Ryckbosch. The triangular model as an instrument for visualising and analysing residuality. Journal of Archaeological Science, 34(4):649 - 655, 2007.

[20] Z. Kulpa. Diagrammatic representation of interval space in proving theorems about interval relations. Reliable Computing, 3(3):209-217, 1997. 